\title{
Defect Evolution in GaAs-based Low-mismatch Heterostructures
}

Abhinandan Gangopadhyay ${ }^{1}$, Aymeric Maros ${ }^{2}$, Nikolai Faleev ${ }^{2}$, Martha R. McCartney ${ }^{3}$ and David J. Smith $^{3}$

1. School for Engineering of Matter, Transport and Energy, Arizona State University, Tempe, AZ, USA

2. School of Electrical, Computer and Energy Engineering, Arizona State University, Tempe, AZ, USA

3. Department of Physics, Arizona State University, Tempe, AZ, USA

Epitaxial growth of thin films of ternary semiconductors such as $\operatorname{In}_{1-x} G_{x} A$ s on GaAs substrates enables band-gap engineering which is very useful for many device applications such as intermediate-band solar cells, lasers, light-emitting diodes, etc. Due to the difference in lattice parameters between film and substrate, the film/substrate interface is subjected to bi-axial misfit strain leading to the introduction of misfit dislocations which are liable to act as non-radiative recombination centers, that are highly deleterious for device performance. Characterization of interfacial defects in these heterostructures is critical for developing a fundamental understanding of strain relaxation processes and eventually finding a path towards minimizing defect density by optimizing growth conditions, film thickness, etc. In this study, transmission electron microscopy (TEM) was used to investigate defect evolution in molecular beam epitaxy-grown low-mismatch (misfit strain $\sim 0.6 \%$ ) $\mathrm{GaAs}_{0.92} \mathrm{Sb}_{0.08} / \mathrm{GaAs}(001)$ and $\mathrm{In}_{0.08} \mathrm{Ga}_{0.92} \mathrm{As} / \mathrm{GaAs}(001)$ heterostructures with film thicknesses in the range of 50 to $4000 \mathrm{~nm}$. All samples had a GaAs capping layer of 50-nm thickness. Plan-view and cross-sectional TEM samples were prepared by polishing, dimpling and liquid-nitrogen argon-ion milling. Philips-FEI CM-200 FEG and JEOL ARM-200F microscopes operated at $200 \mathrm{keV}$ were used for bright-field TEM and aberrationcorrected scanning transmission electron microscopy (STEM) imaging, respectively.

Figure 1(a) shows a representative plan-view TEM image revealing asymmetric distribution of misfit dislocations along orthogonal $<110>$ directions at the film/substrate interface of the heterostructure with $100-\mathrm{nm} \mathrm{GaAs}_{0.92} \mathrm{Sb}_{0.08}$ layer. $\boldsymbol{g} \cdot \boldsymbol{b}$ analysis confirmed that the straight non-periodic misfit dislocations in fig. 1(a) were of $60^{\circ}$ type. The asymmetric distribution of $60^{\circ}$ misfit dislocations at the initial stage of relaxation is often attributed to different mobility of the two types of $60^{\circ}$ dislocations known as $\alpha$ dislocations (As-terminated core) and $\beta$-dislocations (Ga-terminated core). The asymmetry, however, disappears in thicker films when rapid strain relaxation occurs. Figure 1(b) shows misfit dislocation distribution at the film/substrate interface of the heterostructure with 250 -nm-thick $\mathrm{GaAs}_{0.92} \mathrm{Sb}_{0.08}$ layer, which has undergone rapid relaxation. The asymmetric distribution of $60^{\circ}$ dislocations was observed (see fig. 1(c)) at the cap/film interface when the film is sufficiently relaxed to exert sufficient tensile strain to trigger plastic deformation in the capping layer. A simple method was developed to distinguish between the two $<110>$ directions in plan-view orientation, which involved crystal polarity determination by matching experimental convergent beam electron diffraction patterns with simulated patterns in $<110>$ zone-axis orientation. By measuring dislocation spacing from collage of images recorded from an area of over $100 \times 100 \mu \mathrm{m}^{2}$ along the $<110>$ directions, it was found that $\beta$-dislocation density was higher at the compressively-strained film/substrate interface in both $\mathrm{InGaAs} / \mathrm{GaAs}$ and $\mathrm{GaAsSb} / \mathrm{GaAs}$ heterostructures whereas $\alpha$-dislocation density was higher at the tensile-strained cap/film interface. Possible mechanisms explaining this kind of asymmetry reversal will be discussed.

Atomic-resolution high-angle annular-dark-field (HAADF)-STEM imaging was used to obtain information about the atomic arrangement at the defect-cores. All $60^{\circ}$ misfit dislocations at both 
film/substrate and cap/film interface were found to be dissociated. Figure 2(a) shows atomic-resolution HAADF-STEM image of an intrinsic stacking fault (ISF) located at the film/substrate interface of the heterostructure with 1000 -nm-thick $\mathrm{GaAs}_{0.92} \mathrm{Sb}_{0.08}$ layer. Burgers circuit analysis showed that this ISF was created by dissociation of a perfect $60^{\circ}$ dislocation with $\boldsymbol{b}=a / 2[0 \overline{11}]$ and bounded by $90^{\circ}$ partial dislocation with $\boldsymbol{b}=a / 6[1 \overline{12}]$ and $30^{\circ}$ partial dislocation with $\boldsymbol{b}=a / 2[\overline{121}]$ at its two ends. The core of the $30^{\circ}$ partial dislocations in most cases was comprised of an unpaired atomic column. Comparison with structural models (shown in fig. 2(b)) proposed by previous authors [1] led to the conclusion that $60^{\circ}$ misfit dislocations in these systems were predominantly of glide character. In addition to ISFs, atomicresolution images of Lomer edge dislocations and stair-rod dislocations were also obtained. Atomic structure of these dislocation-cores will be discussed in detail. [2]

References:

[1] J. P. Hirth and J. Lothe, Theory of Dislocations, 2nd ed. (Wiley, New York) 1982.

[2] This work was supported by the National Science Foundation and the Department of Energy under NSF CA No. EEC-1041895. The authors acknowledge the use of facilities within the John M. Cowley Center for High Resolution Electron Microscopy at Arizona State University.
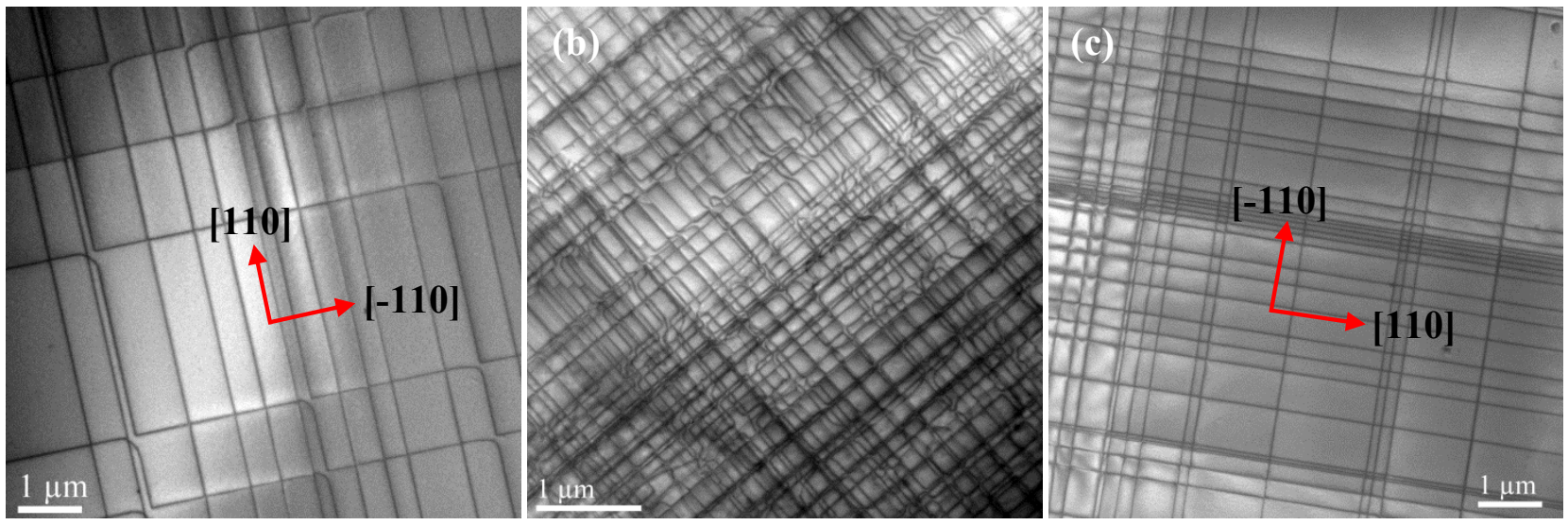

Figure 1. Plan-view $<001>$ TEM images showing distribution of misfit dislocations at the film/substrate interface of heterostructures with (a) 100-nm-thick, and (b) 250-nm-thick $\mathrm{GaAs}_{0.92} \mathrm{Sb}_{0.08}$ layer, and at the cap/film interface of heterostructure with (c) 2000 -nm-thick $\mathrm{GaAs}_{0.92} \mathrm{Sb}_{0.08}$ layer.

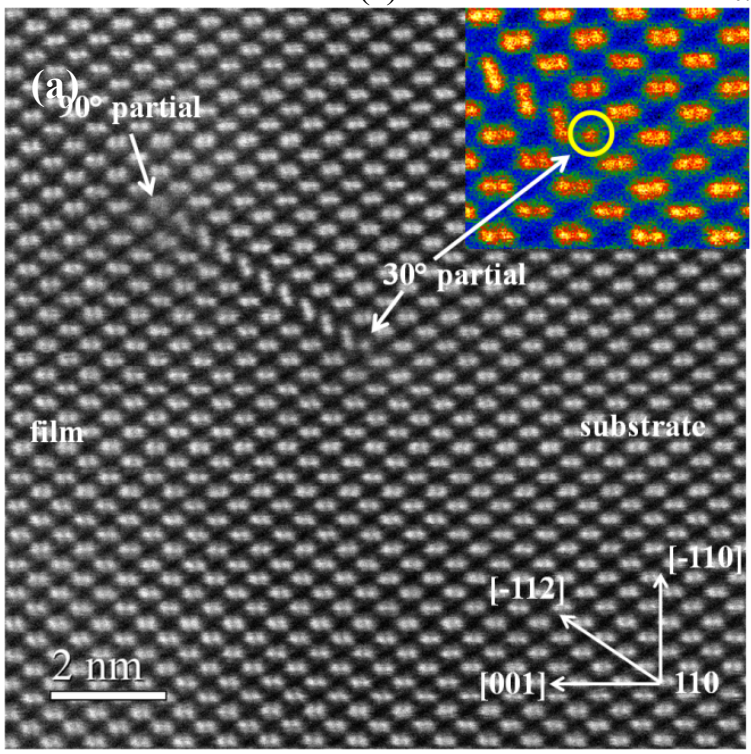

(b)

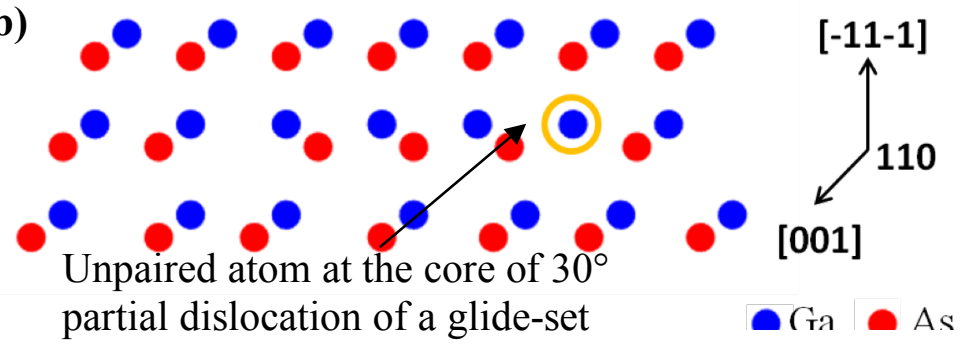
dissociated $60^{\circ}$ dislocation

Figure 2. (a) Aberration-corrected HAADF-STEM image of ISF at $\mathrm{GaAs}_{0.92} \mathrm{Sb}_{0.08} / \mathrm{GaAs}$ interface. The pseudo-colored image in the inset shows a magnified view of $30^{\circ}$ partial core, where the unpaired atomic column is identified with a yellow circle. (b) Matching structural model showing atomic configuration of glide-set dissociated $60^{\circ}$ dislocation. 